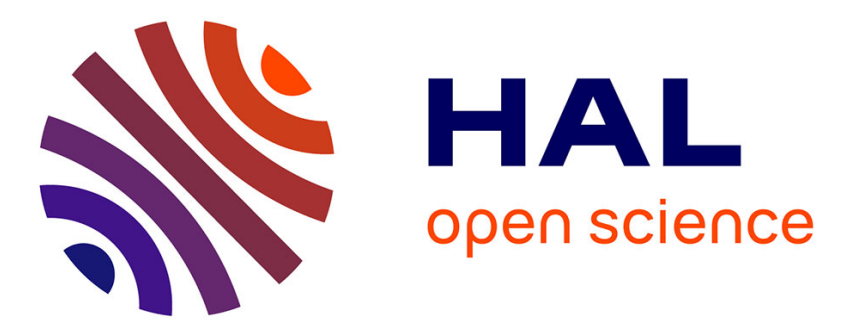

\title{
TUMOR SEGMENTATION FROM PET/CT IMAGES USING LEVEL SETS METHOD
}

Sonia Khatchadourian, Stéphane Lebonvallet, Michel Herbin, Jean-Claude Liehn, Su Ruan

\section{- To cite this version:}

Sonia Khatchadourian, Stéphane Lebonvallet, Michel Herbin, Jean-Claude Liehn, Su Ruan. TUMOR SEGMENTATION FROM PET/CT IMAGES USING LEVEL SETS METHOD. 6th IFAC Symposium on Modeling and Control in Biomedical Systems, Sep 2006, Reims, France. pp.255-260, 10.3182/20060920-3-FR-2912.00048 . hal-02360790

\section{HAL Id: hal-02360790 https://hal.science/hal-02360790}

Submitted on 13 Nov 2019

HAL is a multi-disciplinary open access archive for the deposit and dissemination of scientific research documents, whether they are published or not. The documents may come from teaching and research institutions in France or abroad, or from public or private research centers.
L'archive ouverte pluridisciplinaire HAL, est destinée au dépôt et à la diffusion de documents scientifiques de niveau recherche, publiés ou non, émanant des établissements d'enseignement et de recherche français ou étrangers, des laboratoires publics ou privés. 


\title{
TUMOR SEGMENTATION FROM PET/CT IMAGES USING LEVEL SETS METHOD
}

\author{
Sonia Khatchadourian* Stéphane Lebonvallet* \\ Michel Herbin ${ }^{* *}$ Jean-Claude Liehn ${ }^{* * *}$ Su Ruan* \\ * CReSTIC, 9 rue de Québec, Troyes 10024 \\ ** CReSTIC, rue des Crayères, Reims 51000 \\ *** Institut Godinot, 1 rue du général Koenig, Reims 51056
}

\begin{abstract}
In the framework of detection, diagnostic and treatment planning of tumors, the Positron Emission Tomography (PET) examination coupled with a Computed Tomography (CT) have become recently the most efficient technique. Manual segmentation of regions of interest (ROI) could be time consuming and this is necessary to obtain information about patient pathology. The aim of our study is to propose an automatic solution to this problem. This paper presents an approach of tumor segmentation based on a level set method. One critical step, the algorithm stopping, is automatic. The results obtained by the proposed method dealing with PET-CT images are encouraging. Copyright (C)2006 IFAC
\end{abstract}

Keywords: Image, Partial differential equations, Segmentation

\section{INTRODUCTION}

PET (Positron Emission Tomography) and CT (Computed Tomography) scans are both standard imaging tools that physicians use to pinpoint disease states in the body. A PET scan demonstrates the biological function of the body before anatomical changes take place, while the $\mathrm{CT}$ scan provides information about the body's anatomy such as size, shape and location. By combining these two scanning technologies, a PET/CT scan enables physicians to more accurately diagnose and identify cancer, heart disease and brain disorders, and also to determine how much cancer has spread, and how well cancer treatment is working. Accurate and automatical tumor segmentation is an important issue in many applications of medical image system for quantitative studies. As known, manual tracing by an expert of a tumor in $3 \mathrm{D}$ is not only exceedingly time consuming, but also exhausting for experts. Therefore, an automatical segmentation is necessary.
Many segmentation methods have already been used in medical imaging field. A simple method is the thresholding. (Malyapa et al., 2002) used a binary threshold to segment tumorous areas in the pelvis region. This method allows the user to obtain results very quickly but it is not accurate. The seeded region growing is one of segmentation methods. It consists of choosing seeds, and growing them according to a criteria. (Adams and Bischof, 1994) choose the seeds as the local extrema and then to grow them until the regions touch themselves. But when one need to delineate just one region, one cannot venture to fill the entire image. That is why (Zoroofi et al., 2004) have chosen the seeds upon the histogram and then let grow until the criteria was no longer verified. This work has been used to the segmentation of a non-necrotic femoral head. (Mancas and Gosselin, 2004) proposed a segmentation based on watersheds (Roerdink and Meijster, 2001) to segment PET /CT images. The principle of the watershed technique is to transform the gradient 
of a greyscale image into a topographic surface. (Mancas and Gosselin, 2004) used an iterative watersheds. A very common way to segment medical images is to use an atlas of the human body as a priori knowledge. (Bondiau et al., 2005), (Ehrardt et al., 2001) and (Lorenzo-Valdez et al., 2004) have used such approach respectively on the brain, the hip and the heart atlases. The statistical methods have been also studied to deal with the medical images, because of noise presenting in images. (Ruan et al., 2000) propose a statistical segmentation based on Random Markov Fields to segment brain RM images. The fuzzy segmentation offers an interesting point of view to take into account ambiguities of objects to segment. As shown in (Dou et al., 2002), membership functions are modeled to express the fuzzy signals of the brain tumor observed in different types of images. Among various image segmentation techniques, active contour model [2] has emerged as a powerful tool for semi-automatic object segmentation. The basic idea is to evolve a curve, subject to constraints from a given image, for detecting interesting objects in that image. It consists of the resolution of systems of partial differential equations for which interface propagation phenomenon has to be described. The active contour models are often implemented based on level set method (Sethian, 1999), which is a powerful tool to capture deforming shape. But it has the disadvantage of a heavy computation requirement even using the narrow band evolution. The fast marching method is proposed for monotonically advanced fronts (Sethian, 1999), and is extremely faster than level set evolution. Generally, there are three key problems needed to be solved to implement the curve evolution methods. The first one is the initialization of the seed points. The second one is the formulation of the speed function. And the last one is the determination of the stopping criterion.

The level set methods have been widely applied in medical imagery (Suri et al., 2002) in different domains : the brain (Xie et al., 2005), the bone (Morigi and Sgallari, 2004), the vascular trees (Farag et al., 2004) and so on... The most common way to initialize the level set is the manual selection of a ROI which seems to be relevant ((Xu et al., 2000), (Farag et al., 2004) and (Xie et al., 2005)). Sometimes a simple mouse click combined with a fast marching approach (Fan, n.d.) is used. In this case the final contour determined by the fast marching step is the initial front of the level set. Those methods are semiautomated while we are focused on the automated methods. (Morigi and Sgallari, 2004) proposed an automated method but the imaging system is not the same as the subject of our study.

Our work consists of detecting tumors from the whole body image volume acquired by a PET/CT device. We have no a priori knowledge on the tumor location. A contour evolution model using a level set method with an initialization based on thresholding is proposed in this paper.

The paper is organized as follows. Firstly, an overview of our study is described. Secondly, the level set method and its implementation will be exposed. The different steps of our approach and the associated results are then presented. Finally we will conclude and give some perspectives.

\section{OVERVIEW OF THE STUDY}

\subsection{PET-CT imaging system}

PET/CT combines the functional information from a PET with the anatomical information from a CT into one single examination. Registration between both images is not needed. A PET detects changes in cellular function: how cells are utilizing nutrients like sugar and oxygen. Since these functional changes take place before physical changes occur, PET can provide information that enables physician to make an early diagnosis. A CT scanner uses a combination of x-rays and computers to give the radiologist a non-invasive way to see inside a human body. One advantage of $\mathrm{CT}$ is its ability to rapidly acquire multiple twodimensional image slices of the anatomy.

For giving an optimal treatment, physicians carry out usually manual segmentations to obtain information about sizes, location and image signals of tumors. The objective of our work is to provide a system allowing to automatically segment tumors with a good precision. Since the PET pinpoints increased metabolic activity in cells, tumorous areas can be better observed. Hence, the tumor segmentation is carried out in PET images. Then the segmented regions are projected into CT images to obtain an anatomical localization.

\subsection{Segmentation framework}

Most previous works which have been carried out on the topic of segmentation only considered a part of the body of the patient. Our aim is at detecting the tumorous areas from the whole body. Any a priori knowledge about locations of the tumors are taken into account. As the PET images are usually noisy and bad contrasted, the methods based on the image intensity or gradient are not efficient in these cases. The statistical methods cannot be neither used efficiently due to the small size of the tumor : they are too small to get statistical properties comparing with all images. The solution of the evolving contours is interesting in this case because they can grow to 
the expected size of the tumorous areas with help of geometrical and intrinsic properties.

To segment the $3 \mathrm{D}$ images, we process the $2 \mathrm{D}$ images slice-by-slice. The proposed framework consists of 3 steps: seed detection giving a set of seeds susceptible to belong to the tumor; seed selection allowing to delete aberrant seeds and to consider the left as the initial tumor contours; contour evolution according to an active contour model.

The seed detection consists of finding ROIs using intensity information. The areas of high glucose activity lead to high gray levels observed in PET images. A thresholding of images can be carried out to obtain the ROIs. The problem is how to choose the threshold. As known, the histogram can give the information about the distribution of grey levels. The maximum of the histogram is firstly found, which represents body tissues. Supposing that the number of pixels belonging to tumor regions has less than that of the pique of histogram. The threshold is then defined as the gray level on which the number of pixels equals to the maximum multiplied by a proportionality factor $\alpha$ which is given by experiences.

After the thresholding of images, several seeds are obtained in which some of them do not belong to the tumor. Not only the small regions which could be tumorous, are detected as seeds, but also the big regions representing some anatomical regions (heart for example) which give high intensity. The big regions can be easily moved out from the seeds if their sizes, compared to the size of the patient body, are significant. An erosion, morphological mathematics operator, is carried out to decrease the sizes of seeds. Thus, the initial contours of those plausible ROIs are preserved. This seed selection step allows us to delete aberrant seeds and to keep that of tumorous areas. From the obtained initial contours (seeds), a level set method is used to grow them to find the tumor contours. In the next section, this method is presented in details.

\section{CONTOUR EVOLUTION BASED ON LEVEL SET METHOD}

The level set method has been introduced by (Osher and Sethian, 1988) to solve the partial differential equations. It refers to the theory of the curve evolution. The algorithm proposed by Sethian (Sethian, 1999) has been widely applied to many domains ((Fan, n.d.), (Xu et al., 2000))

\subsection{Theory}

Considering a curve represented by a level set function $\Phi$. For a point $\mathrm{p}, \Phi(p)$ is the signed minimum distance from $\mathrm{p}$ to the contour :
- if $\Phi>0, \mathrm{p}$ is outside the contour,

- if $\Phi=0, p$ is on the contour,

- if $\Phi<0, p$ is inside the contour.

From a geometric point of view, the evolution of a contour can be described as follows :

$$
\frac{\partial x}{\partial t}=V N
$$

with $x$ a point of the contour, $V$ the speed function, $N$ the normal vector of the curve at $x$. The evolution of the curve depends on the normal vector $N$ of the curve and the curvature $K$ at each point of the curve, with :

$$
\begin{gathered}
N=\frac{\nabla \Phi}{|\nabla \Phi|} \\
K=\nabla \frac{\nabla \Phi}{|\nabla \Phi|}=\frac{\Phi_{x x} \Phi_{y}^{2}-2 \Phi_{x} \Phi_{y} \Phi_{x y}+\Phi_{y y} \Phi_{x}^{2}}{\left(\Phi_{x}^{2}+\Phi_{y}^{2}\right)^{3 / 2}}
\end{gathered}
$$

To describe the evolution of the curve we need to define the initial curve as zero level set :

$$
\Phi((x(t)), t=0)=0
$$

To associate the zero level set to the evolving curve at each time and to derive the motion equation for this level set function, the zero level set has to be re-initialized at each time step :

$$
\Phi((x(t)), t)=0
$$

After derivation of the equation 3 :

$$
\Phi_{t}+V \nabla \Phi((x(t)), t)=0
$$

where $V=x^{\prime}(t) \cdot N$ is defined as a speed function and $\nabla \Phi$ is the gradient of the level set function $\Phi$. This speed function is the key of the implementation of the level set method.

\subsection{Speed function}

The speed function $V$ depends on :

- local properties given by local geometrical information (curvature, normal of the curve),

- global properties depending on the form and the position of the front,

- independent properties defined as a fluid velocity that transport passively the front.

Based on these properties, the speed function can be expressed as follows :

$$
V=V_{\text {prop }}+V_{\text {curv }}+V_{a d v}
$$

with $V_{\text {prop }}=V_{0}$ constant speed propagation 


$$
\begin{aligned}
& V_{\text {curv }}=-\epsilon K \text { curvature dependent speed } \\
& V_{a d v}=U(x, y, t) \cdot N \text { advection speed }
\end{aligned}
$$

Since the speed function decreases to zero at the boundary of the area to segment, the components of the speed function are proposed as follows:

$$
\begin{aligned}
\epsilon= & \epsilon_{c} V_{p i j} \text { with } \epsilon_{c} \text { constant }, \\
V_{p i j}= & \frac{1}{1+G_{i j}} \text { with } G_{i j} \text { the image gradient }(7) \\
& \text { at pixel }(i, j), \\
U_{i j}= & \beta \nabla V_{p i j} \text { with } \beta \text { constant . }
\end{aligned}
$$

$\epsilon_{c}$ introduces a viscosity notion around the contour and $\beta$ controls the attraction force. The bigger is $\epsilon_{c}$, the slower is the evolution of the contour. The bigger is $\beta$, the faster is the evolution of the contour. Those parameters are to be defined according to used images. For further details on the implementation of the speed function, see (Sethian, 1999) and (Xu et al., 2000).

The stopping criteria depends on the speed function, therefore indirectly on the intrinsic parameters of the images. The evolution of contours stops when the speed function $V=0$.

\subsection{The narrow band}

The problem of this method is that it takes a long time to compute if the update of the level set function is made on the entire image. The solution proposed by (Chopp, 1993) is to compute the level set function in a narrow band around the front. The level set function is only updated when it reaches the boundary of the narrow band. This narrow band approach can reduce importantly the computing time. It has been used in shape recognition by (Malladi et $a l ., 1994)$ and analyzed by (Adalsteinsson and Sethian, 1995) with success. Therefore, the narrow band is also adopted in our method. The width of the narrow band used is 3 pixels around the contour because of the small size of the tumors.

\section{EXPERIMENTAL RESULTS}

\subsection{Data}

The data, we use for our experimentation, consist of PET-CT image volumes corresponding to three patients who have tumors. The size of the images is of $144 \times 144$ pixels, for a resolution of $7 \mathrm{~mm}$ per pixel for PET images. One volume is composed of about 190 slices. Visually, the tumors observed in images are well segmented, confirmed by hospital experts. One of them is presented in this paper to show the efficiency of our method.

\subsection{Choice of parameters}

Different values of the parameters have been studied and tested before validating these ones : $\alpha=$ 0,0025 for the seed detection, $\epsilon_{c}=0,05$ and $\beta=$ 0,005 for the level set. Those values are chosen according to the images to be dealt with. They can be kept if the images to be treated are acquired from the same PET-CT imaging machine.

\subsection{Segmentation}

Ten PET slices of a patient who has a lung tumor are presented here (figure 1). For a good visualization, the gray levels are inverted. The high activity in glucose regions appears dark. The darker region in the middle of the image is the heart. The muscular tissues are brighter : the arms and the ribcage can be seen as the border of the patient body. The brightest regions represent the lungs. The tumor to detect is on the right part of the image, on the lung area near the ribcage.

The different steps of the segmentation are:

- seed detection (figure 2),

- seed selection (figure 3),

- tumor segmentation (figure 4).

The seed detection (figure 2) allows us to determine ROIs which could be contained by tumorous areas. It is achieved thanks to the gray level information of the entire image volume. We know that higher gray levels represent areas of higher glucose activity and the tumors have abnormal glucose activity. But as foreseen the ROIs obtained are not necessarily tumors. The seed selection can help the decision of seed as explained previously. On the figure 3 , it remains only the plausible seeds to initialize the segmentation.

The level set method is carried out image by image for all the volume from the initialization given by the seed selection. We can see on figure 4 that the tumor is well segmented. Unfortunately, the tumor is not the only region segmented because of the seed detection which is not robust enough.

\section{CONCLUSION AND FUTURE WORKS}

This paper presents a work on the automatic segmentation of tumorous areas from whole body information. The tumors are well segmented even if it remains in the results some false regions. Two possibilities have been evoked to solve this :

- Improving the seed detection by using a multiscale binarization method ((Jolion, 1994) and (Trier and Taxt, 1995)) for example. Indeed the question of seed detection has been briefly considered to test the level set method. 


\section{(a) (2) (2)

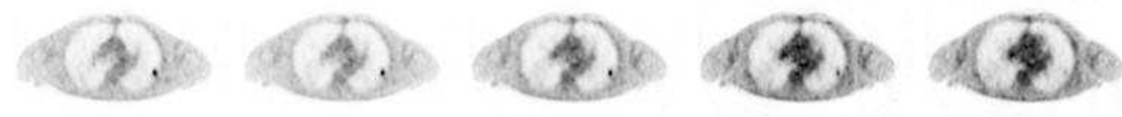

Fig. 1. Patient 3 stack sample illustrating a lung tumor. White matter is the lunge, in darker the muscular tissues, and in dark the heart in the middle and the tumor on the right lung.
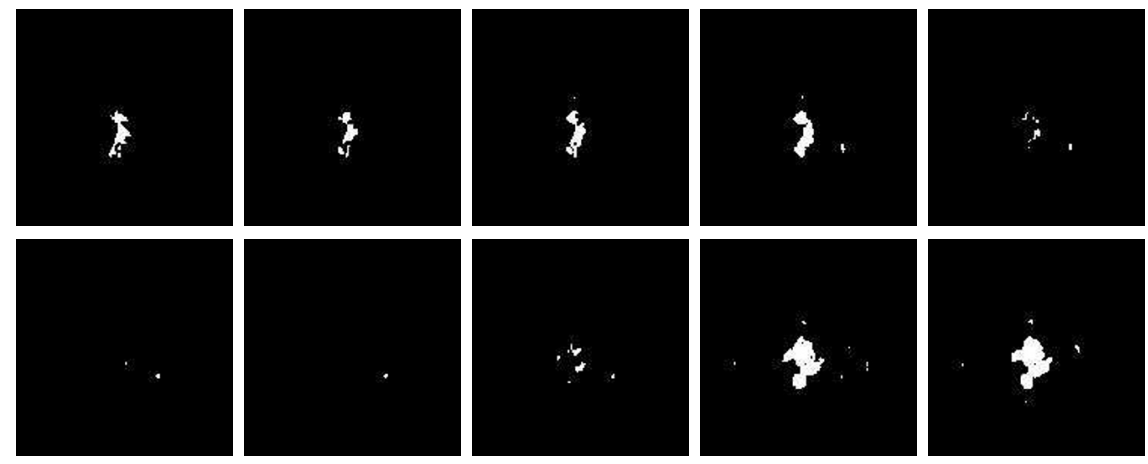

Fig. 2. Seed detection on this sample.
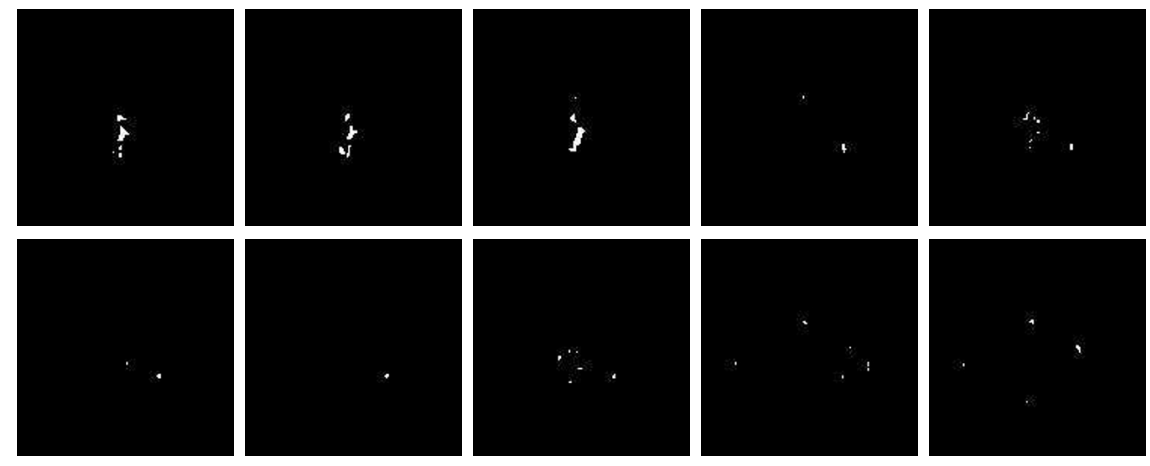

Fig. 3. Seed selection.

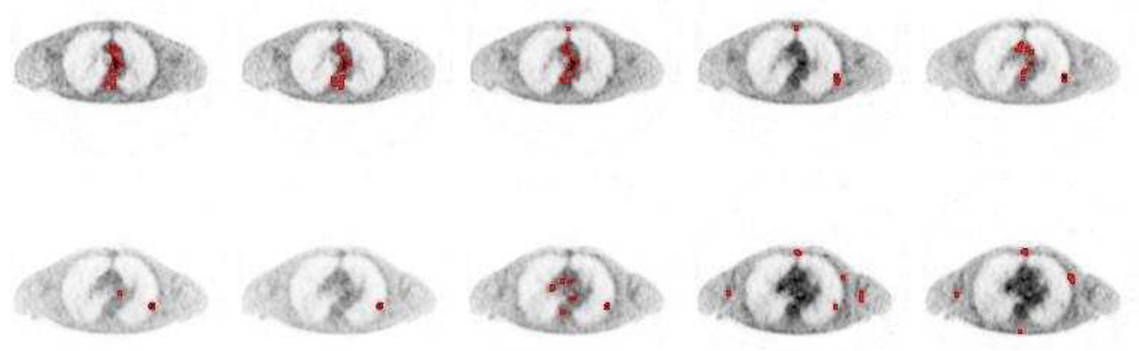

Fig. 4. Level set segmentation. The red contours represent the potential tumorous areas. 
- Implementing a robust classification method to select the tumor contours among all obtained contours. If the initialization step is not robust, the classification step is necessary. A SVM classification, already studied in our laboratory on medical image classification, gave encouraging results (AitAouit, 2004). Our future work is to perform a SVM to obtain only the tumor contours.

Finally the level set should be implemented as a real 3-dimensional method in order to consider the whole 3D information. That allows to improve the performances of the contour evolution.

\section{REFERENCES}

Adalsteinsson, D. and J.A. Sethian (1995). A fast level set method for propagating interfaces. Jour. of Comp. Phys. 118, 269-277.

Adams, R. and L. Bischof (1994). Seeded region growing. IEEE Transaction on Pattern Analysis Machine Intelligence 16, 641-647.

AitAouit, D. (2004). Classification d'images par la mthodes des support vector machines (svm): tude et applications.

Bondiau, P-Y., G. Malandain, S. Chanalet, P-Y. Marcy, J-L. Habrand, F. Fauchon, P. Paquis, A. Courdi, O. Commowick, I. Rutten and N. Ayache (2005). Atlas-based automatic segmentation of mr images: Validation study on brainstem in radiotherapy context. Int. $J$. $R a$ diation Oncology Biol. Phys. 61, 289-298.

Chopp, D.L. (1993). Computing minimal surfaces with level set curvature flow. Jour. of Comp. Phys. 106, 77-91.

Dou, W., Q. Liao, S. Ruan, D. Bloyet, J.M. Constans and Y. Chen (2002). Automatic brain tumor extraction using fuzzy information fusion. Proc. SPIE 4875, 604-609.

Ehrardt, J., H. Handels, T. Malina, B. Strahmann, W. Pltz and S.J. Pppl (2001). Atlasbased segmentation of structures to support virtual planning of hip operations. International Journal of Medical Informatics 64, 439-447.

Fan, D. (n.d.). www.cs.wisc.edu/ ${ }^{\sim}$ fan/levelset/.

Farag, A.A., H. Hassan, R. Falk and S.G. Hushek (2004). 3d volume segmentation of mra data sets using level sets. Academic Radiology 11, 419-435.

Jolion, J-M. (1994). Analyse multirsolution du contraste dans les images numriques. Traitement du Signal 11, 245-255.

Lorenzo-Valdez, M., G.I. Sanchez-Ortiz, A.G. Elkington, R.H. Mohiaddin and D. Rueckert (2004). Segmentation of 4d cardiac mr images using a probabilistic atlas and the em algorithm. Medical Image Analysis 8, 255-265.

Malladi, R., J.A. Sethian and B.C. Vermuri (1994). Evolutionnary fronts for topologyindependent shape modeling and recovery.
Proceedings of Third European Conference on Computer Vision 800, 3-13.

Malyapa, R.S., S. Mutic, D.A. Low, I. Zoberi, W.R. Bosch, R. Laforest, T.R. Miller and P.W. Grigsby (2002). Physiologic fdg-pet three-dimensional brachytherapy treatment planning for cervical cancer. Int. J. Radiation Oncology Biol. Phys. 54, 1140-1146.

Mancas, M. and B. Gosselin (2004). Towards an automatic tumor segmentation using iterative watersheds. Proc. of the Medical Imaging Conference of the International Society for Optical Imaging (SPIE Medical Imaging).

Morigi, S. and F. Sgallari (2004). 3d long bone reconstruction based on level sets. Computerized Medical Imaging and Graphics 28, 377390.

Osher, S. and J.A. Sethian (1988). Fronts propagating with curvature-dependent speed : algorithms based on hamilton-jacobi formulations. J. Computational Physics 79, 12-49.

Roerdink, J.B.T.M. and A. Meijster (2001). The watershed transform: Definitions, algorithms parallelization strategies. Fundamenta Informaticae pp. 187-228.

Ruan, S., C. Jaggi, J. Xue, J. Fadili and D. Bloyet (2000). Brain tissue classification of magnetic resonance images using partial volume modeling. IEEE Transactions on Medical Imaging 19, 1179-1187.

Sethian, J.A. (1999). Level Set Methods and Fast Marching Methods. Cambridge Univerity Press.

Suri, J., K. Liu, S. Singh, S. Laxminarayana and L. Reden (2002). Shape recovery algorithms using level sets in 2-d/3-d medical imagery: A state-of-the-art review. IEEE Transaction on Information Technology in Biomedicine 6, 828.

Trier, O.D. and T. Taxt (1995). Evaluation of binarization methods for document images. IEEE Trans. Pattern Anal. Mach. Intell. 17, 312-315.

Xie, K., J. YANG and Y.M. ZHU (2005). Semiautomated brain tumor and edema segmentation using mri. European Journal of Radiology.

Xu, C., D. L. Pham and J. L. Prince (2000). Medical image segmentation using deformable models. In: Handbook of Medical Imaging - Volume 2: Medical Image Processing and Analysis (J.M. Fitzpatrick and M. Sonka, Eds.). pp. 129-174. SPIE Pres.

Zoroofi, R.A., Y. Sato, T. Nishii, N. Sugano, H. Yoshikawa and S. Tamura (2004). Automated segmentation of necrotic femoral head from 3d mr data. Computerized Medical Imaging and Garphics 28, 267-278. 